\title{
Effect of curcumin nanomicelle on the clinical symptoms of patients with rheumatoid arthritis: A randomized, double-blind,controlled trial
}

\author{
Maryam Javadi1,2 | Hossein Khadem Haghighian2 | Sima Goodarzy2 | Mahnaz Abbasi3 | \\ Marjan Nassiri-Asl4
}

1Children Growth Research Center, Qazvin University of Medical Sciences, Qazvin, Iran

2Department of Nutrition, School of Health, Qazvin University of Medical Sciences, Qazvin, Iran

3Metabolic Diseases Research Center, Qazvin University of Medical Sciences, Qazvin, Iran

4Cellular and Molecular Research Center, Qazvin University of Medical Sciences, Qazvin, Iran

Correspondence

Marjan Nassiri-Asl, Cellular and Molecular Research Center, Department of Pharmacology, Qazvin

University of Medical Sciences, P.O. Box 341197-5981, Qazvin, Iran.

Email: marjannassiriaslm@gmail.com

Mahnaz Abbasi, Metabolic Diseases Research Center, Qazvin University of Medical Sciences, Qazvin, Iran.

Email: dr.mabbasi@yahoo.com

Funding information

Qazvin University of Medical Sciences, Grant/Award Number: IR.QUMS.REC.1396.117

\begin{abstract}
Aim: Rheumatoid arthritis (RA) is a systemic inflammatory disease. In recent years, new drugs with novel targets have been developed to increase the efficacy of drugs in the treatment of RA. Curcumin has shown potent anti-inflammatory effects and is considered an anti-tumor necrosis factor. The present study was conducted to determine the effect of curcumin nanomicelle on the clinical symptoms of patients with RA.

Methods: This randomized, double-blind, controlled trial selected 65 eligible RA patients and randomly divided them into a curcumin nanomicelle group $(n=30)$ and a placebo group $(n=35)$. Curcumin nanomicelle ( $40 \mathrm{mg}$ ) and placebo capsules were administrated to the RA patients 3 times a day for 12 weeks. The Disease Activity Score of 28 joints (DAS-28) and erythrocyte sedimentation rate (ESR) were measured at baseline and after 12 weeks.

Results: The DAS-28, tender joint count (TJC) and swollen joint count (SJC) at baseline and the end of the study were not significant between the curcumin nanomicelle and placebo groups. After the intervention, the within-group DAS-28, TJC and SJC in the curcumin nanomicelle and placebo groups reduced significantly compared to the baseline. The difference in changes between the two groups was not significant. Nonetheless, this change was greater in the case group than in the placebo group. No significant changes were observed in terms of ESR between the two groups of RA patients.
\end{abstract}

Conclusion: Adding curcumin nanomicelle to the RA patients' medication led to some positive changes in the DAS-28, IJC and SJC, although not significantly.

\section{K E YWORDS}

curcumin nanomicelle, disease activity score, rheumatoid arthritis, swollen, tender 